\title{
In situ TEM Studies of Ferroelectric Thin Films
}

\author{
P. Gao, ${ }^{*}$ C. T. Nelson, ${ }^{*}$ J. R. Jokisaari,* S.H. Baek, ${ }^{* *}$ C. B.Eom, ${ }^{* *}$ E. G. Wang, ${ }^{* * *}$ and X. Q. Pan*
}

*Department of Materials Science and Engineering, University of Michigan, Ann Arbor, Michigan 48109

** Department of Materials Science and Engineering, University of Wisconsin-Madison, Madison, Wisconsin 53706

*** School of Physics, Peking University, Beijing 100871, P. R. China

Ferroelectrics such as lead zirconate titanate (PZT) are attractive for applications in nanoelectronics including high-density and non-volatile memories [1]. The polarization of this perovskite family of ferroelectric materials results from the offset of the metallic cations and oxygen anions in the unit cell. Applications such as memory require reliable switching between polarization states through the application of an external electrical field which favors a single polarization state. Such ferroelectric switching occurs by nucleation and growth of favorably oriented domains [2], a heterogenous process which is dominated by nanoscale defects and interfaces. Understanding the kinetics of this process is vital for the performance of such devices.

Here we report real-time studies of polarization switching of PZT thin films by in-situ transmission electron microscopy (TEM). By this approach the time varying domain structure is characterized at nanometer and millisecond spatial and temporal resolution using a probe which is independent from the electrical bias. For this work we use $100 \mathrm{~nm}$ tetragonal $(001) \mathrm{Pb}\left(\mathrm{Zr}_{0.2} \mathrm{Ti}_{0.8}\right) \mathrm{O}_{3}(\mathrm{PZT})$ films grown on (110)- $\mathrm{DyScO}_{3}$ substrates buffered by a $50-\mathrm{nm} \mathrm{SrRuO}_{3}$ (SRO) conducting electrode. The film was switched by applying a bias in a planar capacitor type structure between a freely positionable tungsten surface probe and the SRO epitaxial bottom electrode (Fig 1a).

The as-grown film was c-axis oriented with a predominant upward polarization. Fig. 1 shows a chronological series of the polarization reversal beneath the probe as the applied bias is linearly increased from $0 \mathrm{~V}$ to $+9 \mathrm{~V}$ with respect to the grounded metallic SRO counter electrode. A small downward poled domain nucleates at the top surface between $2.9 \mathrm{~V}$ and $3 \mathrm{~V}$, visible as a dark region in the diffraction contrast TEM image Fig. 1c. With increasing bias \& time the nucleated domain quickly propagates forward until it reaches the bottom electrode (Fig. 1d-g) followed by slow lateral growth (Figs.1h-k). After removal of the bias the new domain remains essentially unchanged (Fig.1l). Under reverse bias the newly formed domains back-switch to the original state by the slow reduction of the domain volume and/or by the nucleation of new domains. Because the polarization is proportional to the domain volume we can construct polarization-electric field (P-E) hysteresis loops corresponding to this voltage cycle (Fig. 2).

In this study we find the nucleation of up and downward poled domains is dominated by opposing film surfaces due to the built-in electric fields created by the formation of Schottky junctions. Such real-time observation provides a fundamental understanding of ferroelectric switching, especially the roles of interfaces and defects in domain nucleation and mobility.

References

[1] O. Auciello et al., Physics Today 51 (1998) 22. 
[2] M. Dawber et al., Reviews of Modern Physics 77 (2005) 1083.

[3] The authors gratefully acknowledge the financial support through DOE grant DoE/BES DEFG02-07ER46416, NSF DMR-0907191 and DOE grant DE-AC02-05CH11231 (NCEM user facilities)
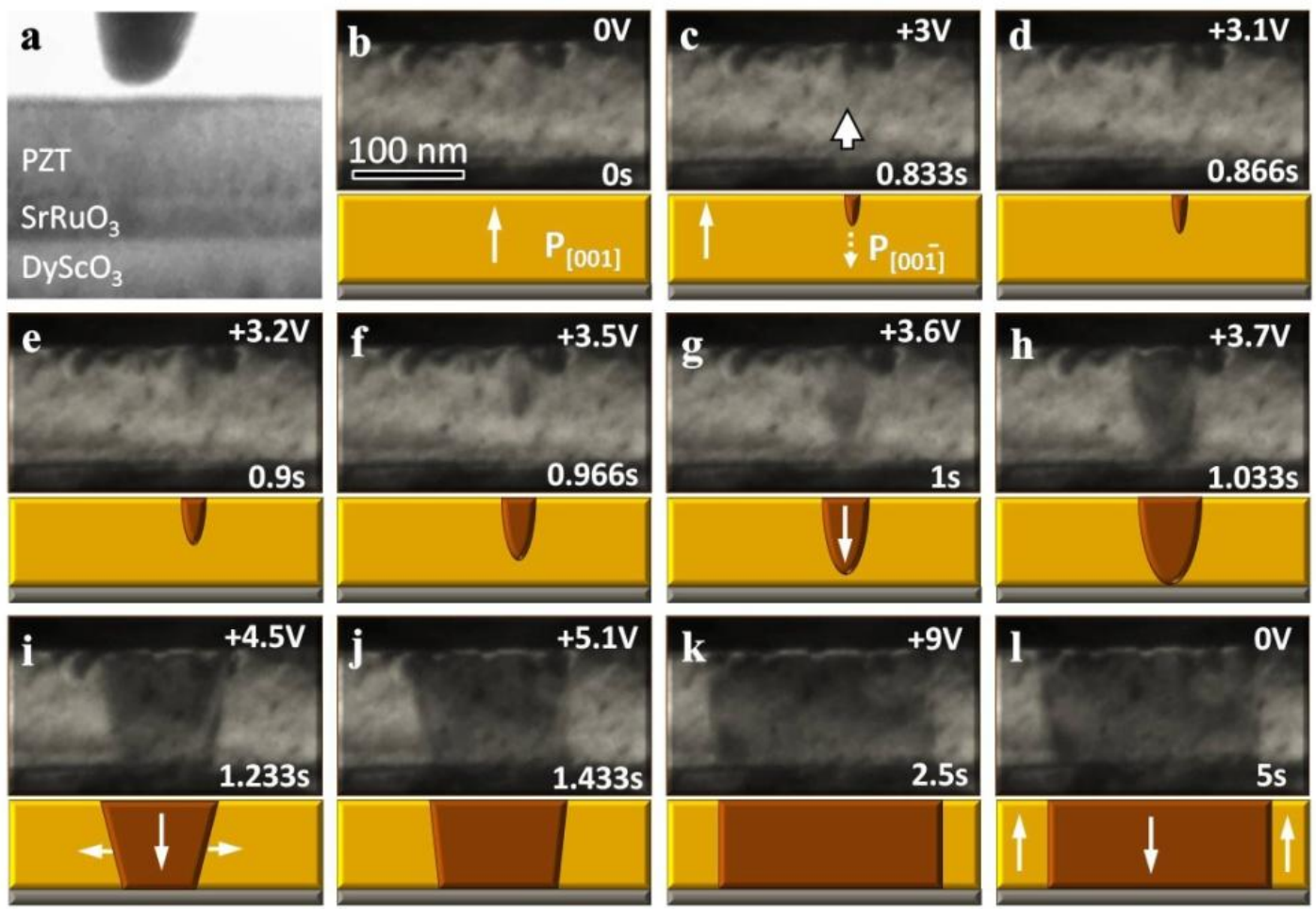

FIG. 1. Image sequence of the domain evolution in a PZT film switched under an increasing bias, 0 $\mathrm{V}$ to $+9 \mathrm{~V}$. The initial domain has an upward polarization direction (b). A downward poled domain nucleates at a bias around $3 \mathrm{~V}(\mathbf{c})$, followed by the forward propagation of domains across the film (d)-(g), and (h)-(k) then widening of the domain.

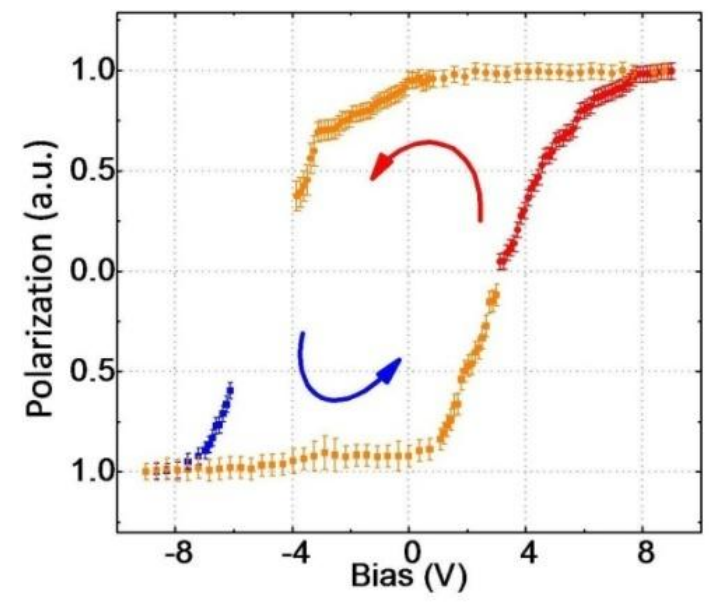

FIG. 2. The polarization calculated by the volume of the switched domain as a function of the applied bias. Upper half: The switching of an upwardly polarized domain and its back switching. Lower half: The switching of a downwardly polarized domain and its back switching. 